\title{
Innovative hybrid pile oscillator technique in the Minerve reactor: open loop vs. closed loop
}

\author{
Benoit Geslot, Adrien Gruel, Stéphane Bréaud, Pierre Leconte, and Patrick Blaise
}

\begin{abstract}
Pile oscillator techniques are powerful methods to measure small reactivity worth of isotopes of interest for nuclear data improvement. This kind of experiments has long been implemented in the Mineve experimental reactor, operated by CEA Cadarache. A hybrid technique, mixing reactivity worth estimation and measurement of small changes around test samples is presented here. It was made possible after the development of high sensitivity miniature fission chambers introduced next to the irradiation channel. A test campaign, called MAESTRO-SL, took place in 2015. Its objective was to assess the feasibility of the hybrid method and investigate the possibility to separate mixed neutron effects, such as fission/capture or scattering/capture. Experimental results are presented and discussed in this paper, which focus on comparing two measurements setups, one using a power control system (closed loop) and another one where the power is free to drift (open loop). First, it is demonstrated that open loop is equivalent to closed loop. Uncertainty management and methods reproducibility are discussed. Second, results show that measuring the flux depression around oscillated samples provides valuable information regarding partial neutron cross sections. The technique is found to be very sensitive to the capture cross section at the expense of scattering, making it very useful to measure small capture effects of highly scattering samples.
\end{abstract}

Index Terms-Neutron cross section measurement; hybrid pile oscillator; minerve experimental reactor.

\section{INTRODUCTION}

$\mathrm{P}$ ILE oscillator techniques are powerful methods to measure small reactivity worth of isotopes of interest for nuclear data improvement [1-3]. The principle is to create, in a well-known neutron flux, a weak and localized perturbation, the characteristics of which (amplitude, phase, energy spectrum) can be connected to some physical parameters (i.e. cross sections). Pile oscillator techniques are called "open loop" if the reactor power is free to drift during the measurement. On the contrary, it is called closed loop when a power control system is activated. Such pile oscillator experiments have long been held in the Minerve facility, operated by CEA at Cadarache [4]. A closed loop reactivity

B. Geslot is with CEA DEN/Cad/DER/SPEx/LPE, Cadarache, Bat.238, F13108, Saint Paul Lez Durance (e-mail: benoit.geslot@cea.fr).

A. Gruel and P. Blaise are with CEA DEN/Cad/DER/SPEx/LPE, Cadarache, Bat.238, F-13108, Saint Paul Lez Durance.

S. Bréaud is with CEA DEN/Cad/DER/SPEx/LDCI, Cadarache, Bat.238, F-13108, Saint Paul Lez Durance.

P. Leconte is with CEA DEN/Cad/DER/SPRC/LEPh, Cadarache, Bat.238, F-13108, Saint Paul Lez Durance. oscillator method is implemented based on measuring the global reactivity perturbation associated to samples motion in the core center. The technique was recently extended by introducing CEA-made high sensitivity miniature fission chambers (MFCs) close to the irradiation channel in order to measure local perturbations of the neutron flux. New detectors and associated acquisition systems were developed by CEA for that purpose [5].

A measurement campaign, called MAESTRO-SL (in which SL stands for "local signal"), took place in 2015 in the Minerve reactor, in a core configuration close to the one used for the MAESTRO oscillation campaign [6-7]. Its objective was to assess the feasibility of measuring local perturbation and, then, to investigate on unfolding capture and scattering cross sections. Closed loop experiments were first performed to provide reference results. Then, open loop measurements were done on a subset of samples.

\section{EXPERIMENTAL SETUP}

Minerve is a pool type reactor operated at low power ( $100 \mathrm{~W}$ maximum). At the center of the core, an irradiation channel is used to introduce various test samples in the core. Samples are held in an oscillator device used to perform pile oscillator experiments. Their reactivity worth (in the range of a few pcm) are obtained and compared against reference materials (e.g. gold, boron, lithium)

In the MAESTRO-SL configuration, four detector locations (8.5 $\mathrm{mm}$ in diameter) were accommodated close to the oscillation channel (13.4 $\mathrm{mm}$ in diameter) in a guiding device made of aluminum alloy. Four MFCs loaded with enriched uranium deposits (Fig. 1) were used to monitor the thermal flux in the central channel vicinity.

A boron chamber, operated in current mode, was used to monitor the reactor power during experiments and to provide a signal proportional to the global perturbation introduced by oscillated samples. This detector was located in the core reflector, far enough from the core center so that its signal is proportional to the reactor global fission rate.

The power control system (PCS) is based on a rotary cadmium control rod (the so-called "pilot rod") located in the reflector, next to the driver zone. Reactivity is ajusted by changing the angle between rotor and stator, which varies the active cadmium surface. A retroaction loop fed by the boron chamber signal is used to stabilize reactor power. 


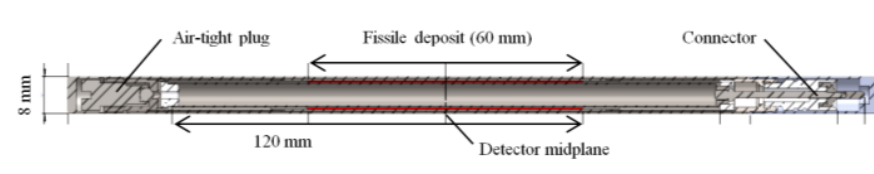

Fig. 1. Drawing of the CF8R-lg fission chamber (dimensions are in $\mathrm{mm}$ ).

Oscillated samples were made of a column of material of interest (MOI), sometimes diluted into a matrix such as water, and enclosed in a cladding sheath (aluminum, Zircaloy-4 or both). Most samples contained scattering materials (e.g. D2O, $\mathrm{Be}$ ) or neutron absorbing materials (e.g. acid boric solutions, gold wires). Two samples contained fissile material $\left(\mathrm{UO}_{2}\right.$ pellets at $0.25 \%$ and $3 \%$ enrichment in ${ }^{235} \mathrm{U}$ ).

\section{DESCRIPTION OF EXPERIMENTS}

In CLO experiments, the global signal, denoted as $S_{G}(t)$, was issued by the angular sensor that monitored the pilot rod motion. Thanks to the reactivity retro-action loop, all detectors recorded pseudo-square waveforms very similar in shape to the one of the sample motion in and out of the core (trapezoidal), as shown on Fig. 2. Both local and global signals were first rescaled by dividing by their average. Their amplitudes are respectively denoted $\Delta L$ and $\Delta G$.

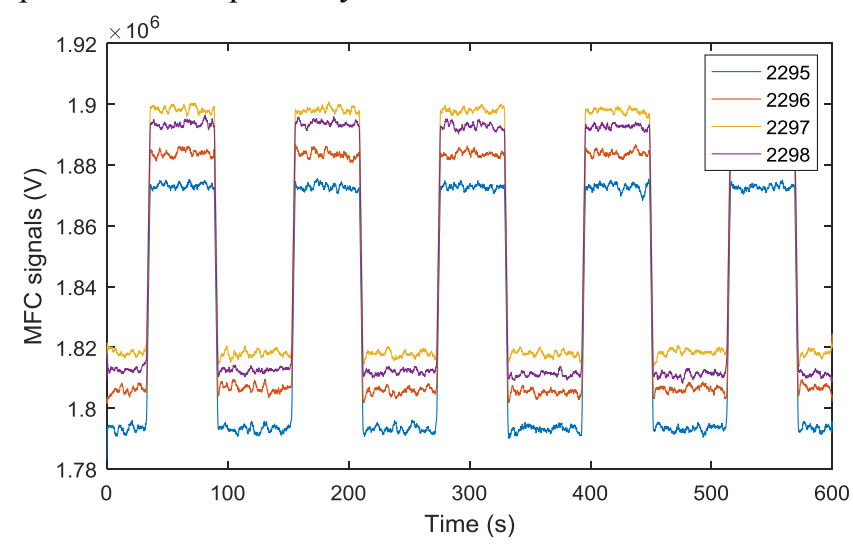

Fig. 2. Signals from MFCs during CL oscillation of $\mathrm{D}_{2} \mathrm{O}$ sample.

In OLO experiments, reactor power was free to evolve along with the oscillator motion. Global signal $S_{G}$ was taken from a boron chamber located in the core reflector. During an oscillation, $S_{G}$ exhibited increasing and decreasing transients driven by delayed neutron precursors.

As for CLO experiments, local signal $S_{L}$ was obtained from local MFCs counting rate. In this case, $S_{L}$ is a mix of a global component driven by the reactor power and a local component proportional to thermal flux changes (in amplitude and spectrum) around the sample.

OLO data processing required first to correct for slow power drifts during experiments. Then, signals were normalized to their average. This way, it is straightforward to derive the local perturbation by subtracting the global component to the local signal, as follows:

$L(t)=\frac{S_{L}(t)}{\bar{S}_{L}}-\frac{S_{G}(t)}{\bar{S}_{G}}$,

As illustrated on Fig. 3, $L(t)$ is a square signal, that can be compared to CLO signal. Amplitude of $L(t)$ is denoted $\Delta L$.
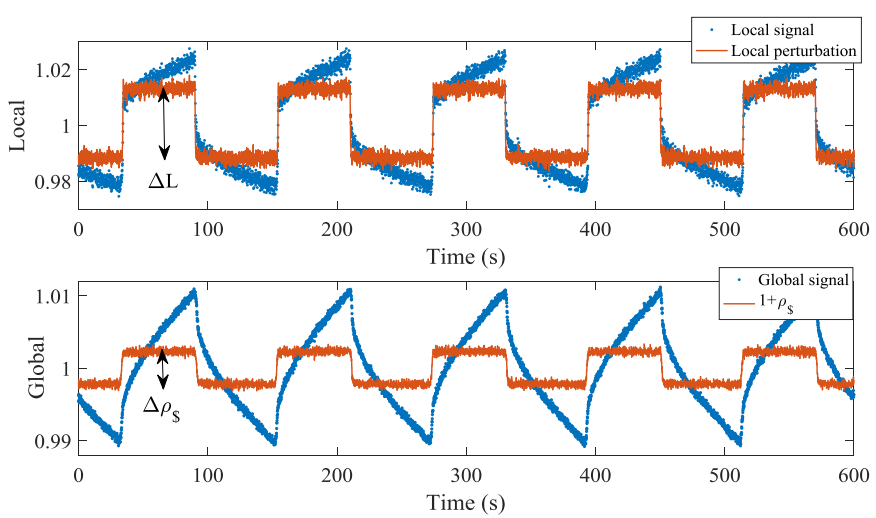

Fig. 3. Local and global signals recorded during the OLO of $\mathrm{D}_{2} \mathrm{O}$ sample.

Analysis of $S_{G}(t)$ can be done by Fourier Series analysis. Because it is a non-square signal, information is contained in the phase as well as in the amplitude of the signal harmonics. Let $T F\left[S_{G}\right](f)$ be the Fourier transform of signal $S(t), f_{0}$ be the oscillation frequency and $T$ the oscillation duration. The modulus $\left|S_{G}\right|_{n}$ of the $n^{\text {th }}$ harmonics is expressed as:

$$
\left|S_{G}\right|_{n}=\frac{1}{T}\left|\operatorname{TF}\left[S_{G}\right]\left(n \cdot f_{0}\right)\right|,
$$

\section{RESULTS AND DISCUSSION}

In order to compare CL and OL results, it was found necessary to rescale OLO results. As expected, global signals had different units because they came from different detectors. A linear fit was applied then to obtain a 2.65 coefficient between angular captor and boron chamber signals $\left(\mathrm{R}^{2}\right.$ coefficient was 0.99995). Unexpectedly, a systematic sensitivity shift between OLO and CLO was observed on inlocal signals. This was a slight difference $(2.5 \%)$, that probably came from a bias introduced by the normalization step, due to the fact that signals are not exactly symmetrical. A correction was made by applying linear fit to correct OLO data $\left(\mathrm{R}^{2}=0.99989\right)$. After rescaling, OLO and CLO data were found to be in very good agreement.

In the past, local oscillation experiments were performed to separate effects from different cross sections. With adequate calibration procedure, it was possible to measure at the same time fission and radiative capture cross sections in fissile materials [8]. Another interesting experiment was the measurement of very small capture cross sections in strongly scattering materials [9]. Here, independent measurements of both local and global perturbations is achieved. Such hybrid experiment should provide additional information on sample cross sections.

On Fig. 4, results from the whole CLO campaign are plotted in a $2 \mathrm{D}$ space using $\Delta L$ and $\Delta G$ as horizontal and vertical coordinates. 121 oscillation results are represented as dots whereas circles feature averaged results per sample.

A clear linear behavior can be seen for samples of the same type. Straight lines drawn on the graph indicate directions corresponding to neutron effects such as radiative capture in gold (green line), scattering (blue line) and neutron production (red line). 


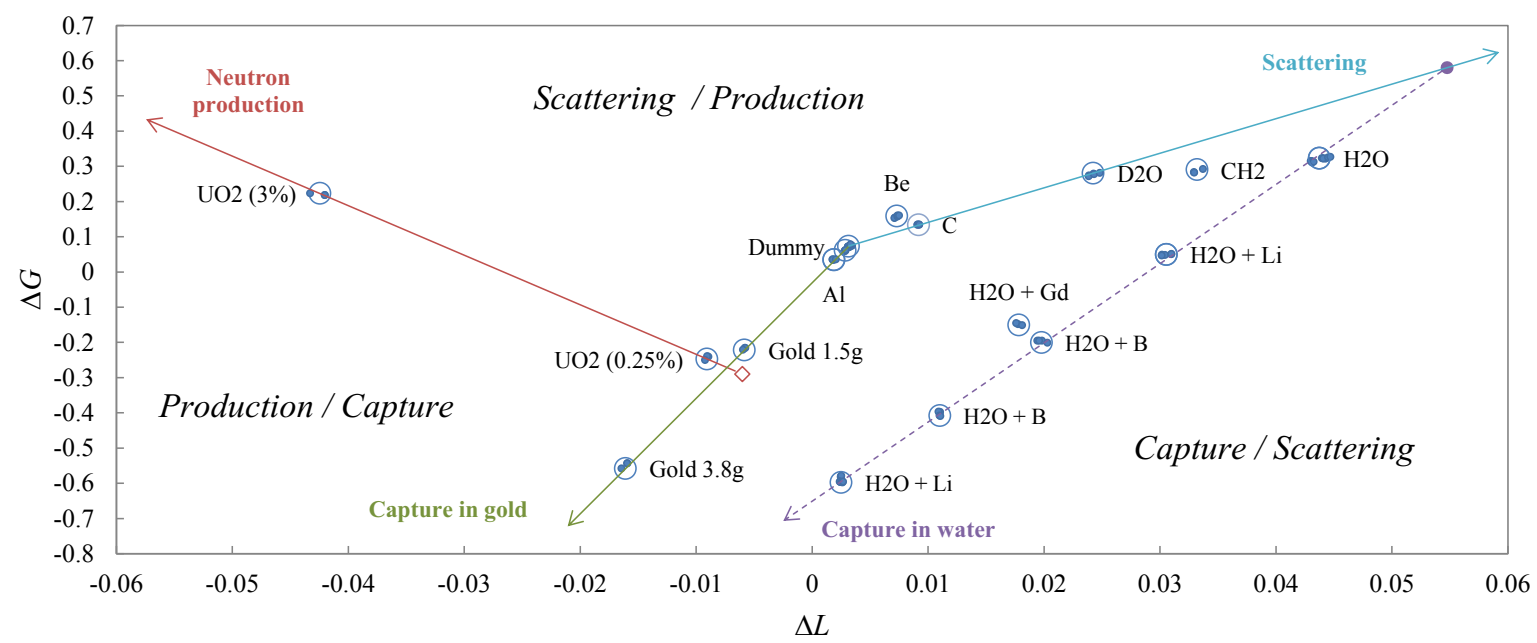

Fig. 4. CLO results in $(\Delta G, \Delta L)$ plane. Dots are measurements and circles are averaged results per sample.

Samples coordinates on the graph are related to the macroscopic cross sections of their components (cladding, matrix and MOI). Let a vector $\boldsymbol{D T}$ be defined by a dummy sample $D$ and a test sample $T$. Its norm $\|\boldsymbol{D} \boldsymbol{T}\|$ is a quantity proportional to the test sample MOI macroscopic cross sections. The proportionality coefficient is complex to determine as it is strongly dependent on the neutron flux shape and spectrum in the sample's vicinity. It is still possible to decompose vector $\boldsymbol{D T}$ into components inside a coordinate space given by two directed lines. Those lines can be graded using reference samples of known composition. Samples components could then be expressed in physical units (mass of reference material or cross sections in barns).

The plane $(\Delta G, \Delta L)$ can thus be divided into separate areas corresponding to mixed neutron effects, such as production/capture area (for fissile sample) or capture/scattering area (for water based samples). Notice that capture and neutron production lines are nearly perpendicular, which is in favor of an optimum separation of the two effects. Separation is less favorable for capture and scattering.

\section{CONCLUSION AND OUTLOOKS}

Pile oscillator techniques are powerful methods to measure small reactivity worth of isotopes of interest for nuclear data improvement. Recently, hybrid pile oscillation experiments (i.e. mixing local and global signals) were carried out in the Minerve facility using newly designed CEA-made high sensitivity miniature fission chambers.

The coupled analysis of samples reactivity effect (global signal) and thermal flux depression surrounding the samples (local signal) allows separating effects into components: fission and capture for fissile samples, scattering and capture for other samples.

Experiments demonstrated the feasibility of measuring in Minerve thermal flux changes as low as $0.1 \%$. Because local flux variations are small and rapidly fading away off samples, a major drawback of the technique is to be rather sensitive to small geometry differences among samples. Adapted sample design and careful experimental setup should mitigate the impact on results quality.
Experimental results show clearly that separating fissile and capture effects is optimum in Minerve setup. A fair separation of scattering and capture effect, although less favorable, achievable.

A comprehensive analysis of the whole results using newly developed capabilities in the TRIPOLI-4 Monte Carlo code should start soon.

\section{ACKNOWLEGEMENTS}

This work was conducted by CEA/Cadarache in the framework of the I3P collaboration regrouping CEA, EdF and AREVA.

\section{REFERENCES}

[1] W. K. Foell, "Small-sample reactivity measure-ments in nuclear reactors". American Nuclear Society, Hinsdale, IL, USA, 1972.

[2] J. I. Hoover et al., "Measurement of Neutron Absorption Cross Sections with a Pile Oscillator," Phys. Rev., vol. 74, no. 8, pp. 864-870, Oct. 1948, 10.1103/PhysRev.74.864

[3] A. L. Dekker and H. R. Kleijn, "The local pile oscillator as a device for measuring epithermal neutron absorption," Nucl. Instr. Meth., vol. 55, pp. 87-92, Jan. 1967.

[4] M. Antony, et al., "Oscillation experiments techniques in CEA MINERVE experimental reactor," $1^{\text {st }}$ ANIMMA Int. Conference, Marseille, June, 2009.

[5] B. Geslot, et al., "A hybrid pile oscillator experiment in the Minerve reactor". Annals of Nuclear Energy, 108, pp. 268-276, 2017.

[6] P. Leconte, et al., "MAESTRO: An ambitious experimental programme for the improvement of nuclear data of structural, detection, moderating and absorbing materials - First results for ${ }^{n a t} \mathrm{~V},{ }^{55} \mathrm{Mn},{ }^{59} \mathrm{Co}$ and ${ }^{103} \mathrm{Rh}$," in ANIMMA 2013 Int. Conf., Marseille, France, 2013.

[7] P. Leconte, et al., "Thermal neutron activation experiments on Ag, In, Cs, Eu, V, Mo, Zn, Sn and $\mathrm{Zr}$ in the MINERVE facility," in WONDER 2015, Aix-en-Provence, 2015, vol. 111.

[8] B. Laponche, M. Brunet, and Y. Bouedo, "Oscillation of Fissile Materials in Thermal-Neutron Critical Assemblies: The Equivalent Sample Method," Nucl. Sci. Eng., vol 48, no. 3, Jan. 1972.

[9] A. Ertaud, V. Raievski, and D. Breton, "Méthode d'oscillation dans la pile, appliquée à la comparaison d'échantillons de graphite," J. Phys. Radium, vol. 12, no. 8, pp. 784-788, 1951. 